\title{
Liquid crystal modulator in the channel of a laser projection microscope
}

\author{
P. Oleksenko, V. Sorokin, R. Zelinskyy \\ V. Lashkaryov Institute of Semiconductor Physics, NAS of Ukraine, 41, prospect Nauky, 03028 Kyiv, Ukraine
}

\begin{abstract}
Using the light modulator based on electrically controlled reflection in the interface between isotropic dielectric and nematic liquid crystal has been viewed. The optical scheme of a laser projection microscope (LPM) with a liquid crystal modulator (LCM) in treatment, observation and recording channels has been proposed, implemented and investigated. In the proposed scheme, LCM can act as a light beam splitter, attenuator and modulator simultaneously. Possibilities of some thin film materials processing by LPM with LCM in the treatment, observation and recording channels are demonstrated.
\end{abstract}

Keywords: liquid crystal, modulator, optical scheme, super radiant laser medium.

Paper received 04.06.04; accepted for publication 21.10.04.

\section{Introduction}

The appearance of laser techniques was initiated by microelectronic requirements. It turned out that laser techniques enable to solve such tasks as adjustment of hybrid microcircuits, quartz resonators and surface acoustic wave device parameters, correction of defects in chips when checking their quality, photomasks in chip production, etc. Laser technique has a lot of advantages in the following fields: small zone of thermal influence, adjustment of treatment depth, high productivity, possibility of treatment at various stages of chip production and after them.

Some articles were devoted to investigations of laser techniques and ways of their implementation $[1,2]$. The technique for simultaneous treatment, registration and observation based on super radiant laser medium is a field of special interest.

For the first time, the idea to obtain image with amplified brightness by using laser active medium was proclaimed in [3]. Later in [4], the practical scheme of LPM was described, and its characteristics were published. In the following articles, a lot of possible schemes of LPM where active medium was used not only for imaging in a screen, but also for simultaneous treatment and observation of objects [5], have been proposed. One of such schemes was implemented in the laser microprojector 09 VP-1000-02. Main characteristics of this laser microprojector were reported in [6]. It is necessary to notice that such type of microprojectors can be successfully used for treatment of thin film electron devices and equipment: photomasks, resistors, semiconductor structures, etc.

High frequency of laser pulses $(8 \mathrm{kHz})$ in a copper vapor laser causes considerable difficulties during the execution of precision technological operations. In the course of the laser treatment, it is necessary to have a possibility of fast laser beam switching (switch on and off) with frequencies comparable to the frequency of laser pulses. In a correspondence with this requirement, investigations of optical emission LCM based on electrically controlled reflection of the light on interface between glass and nematic liquid crystal as an optical element of LPM have been performed [7]. The main goal of this paper was to research LPM characteristics that can be achieved using LCM in observation, recording and treatment channels and its comparison with LPM characteristics without LCM. Main characteristics of LPM with LCM in observation and treatment channels were also investigated.

\section{Peculiar properties of LPM schemes with LCM}

An optical scheme of device for observation, recording and treatment of objects is shown on Fig. 1. Optical scheme of device for observation, recording and treatment of objects with LCM in observation and recording channels is shown in Fig. 2. It consists of super radiant laser medium $l$ with the optical focusing system 2 and 


\section{P. Oleksenko et al.: Liquid crystal modulator in the channel of a laser projection microscope}

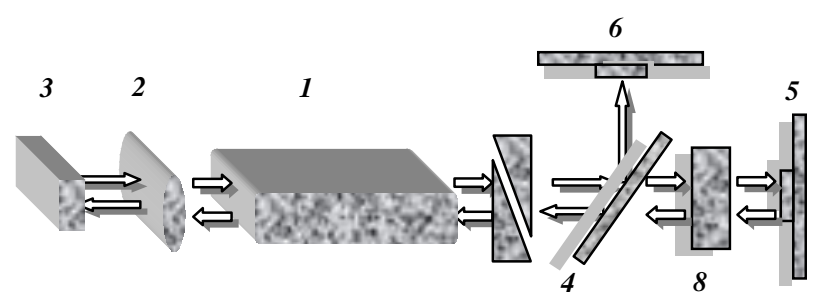

Fig. 1. Optical scheme of the device for treatment, observation and recording objects by using superradiant laser medium: 1 superradiant laser medium, 2 - optical focusing system, 3 - object, 4 - light split mirror, 5 - reflective mirror, 6 - screen, 7 system of optical wedges, 8 - modulator.

object 3 on the one side. On another side of super radiant laser medium 1 placed are LCM 4 and the reflective element 5 . In the plane $n_{1}$, which is conjugated with the plane $n_{2}$, containing the object 3 , located a recording medium 6 , for example, screen for visual observation. Object 3 is treated and observed by super radiant laser medium 1 that can be gas discharge plasma in vapors of copper, gold, barium, etc. A thyratron generator can accomplish excitation of superradiant laser medium. The light beam from super radiant laser medium is collected at the object 3 by the optical focusing system 2 .

It is necessary to notice that in the same time the light beam falls on the recording medium 6 . The light beam reflected by the object 3 is returned through the optical focusing system 2 in superradiant laser medium $l$ where it is amplified. After that, it comes into LCM light splitter where it is divided into two parts. One part of the light beam comes onto the recording medium 6 in order to obtain an amplified brightness and magnified image of the object 3. Another part of the light beam is reflected from the element 5 and returns into the superradiant laser medium 1 and falls onto the object 3 through optical focusing system 2 .

The object 3 , optical focusing system 2 , superradiant laser medium 1, LCM light splitter 4 and recording me-

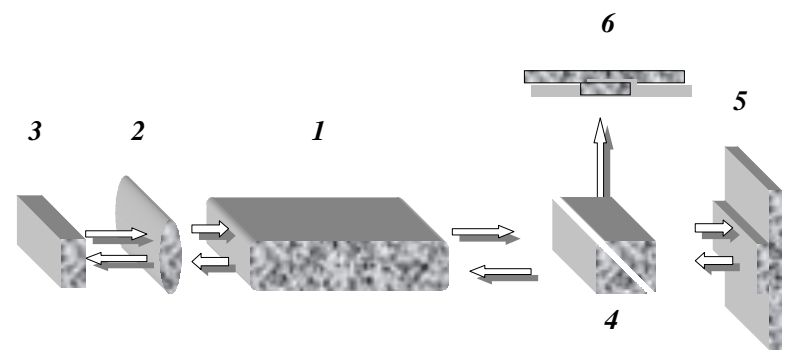

Fig. 2. Optical scheme of device for treatment, observation and recording objects by usingsuperradiant laser medium with LCM in treatment and observation channels: $I$ - superradiant laser medium, 2 - optical focusing system, 3 - object, 4 - LCM, 5 reflective mirror, $6-$ screen. dium 6 located on the same optical axis form an observing channel. Respectively, object 3, optical focusing system 2 , superradiant laser medium 1 , LCM light splitter 4 and reflective element 5 situated at the same optical axis form the treatment channel. At the same time, LCM works as alight splitter and optical shutter. It allows us to acquire necessary object characteristics at the right time moment.

The increase in treatment accuracy and quality, and obtaining light spot with minimum size for the given optical focusing system 2 has been achieved because the dimensions of reflective area of the element 5 in LPM scheme have the same value as the optical scattering spot for the optical focusing system 2 in the plane optically conjugated with the plane containing object 3 . This aim can be achieved in LPM with LCM shown in Fig. 2, if one of LCM control electrodes has a size no more than the size of the scattering spot for the optical focusing system 2. If one of LCM control electrodes has $\mathrm{N}$ control electrodes that it is possible to treat fixed relatively to the optical focusing system 2 object 3 in field of vision of the optical focusing system 2 by the light spot with a limited size. The larger accuracy can be achieved when control electrodes of LCM are realized as a matrix. It is connected with the fact that the scale of movement of the light spot reflected from mirror 5 is proportional to magnification of the optical focusing system 2 .

For some technological tasks, for example, the treatment of object with high selectivity or a treatment of only a part of object in LPM with the scheme shown in Fig. 1 between the light splitter and light shutter placed are the special device that can fluently attenuate light intensity. This device is the movable optical wedge from neutral gray glass.

In LPM with LCM that is represented in Fig. 2, the fluent attenuation of the light intensity is achieved by the change of control voltage peak value applied to LCM electrodes. In this case, the selectivity of treatment is sufficiently higher, because it is possible to treat the object 3 by limited light pulses with a required intensity as it will be shown below.

In the proposed scheme of LPM with LCM for treatment, recording and observation of objects by superradiant laser medium, it is possible to use different types of intensity and movement sensors in order to automate the treatment process like that in [6].

\section{Experimental setup}

According to the scheme shown in Fig. 2, the experimental equipment for treatment, recording and observation of objects by superradiant laser medium was created, and its main parameters and characteristics were measured and investigated.

In experimental tests, LC modulators based on the effect of electrically-controlled light reflection at the interface of a glass and nematic LC were used. Here, the geometry of NLC optical axis reorientation described in [8] was used. Experimental samples of LC modulators 


\section{P. Oleksenko et al.: Liquid crystal modulator in the channel of a laser projection microscope}

were composed of two glass prisms with the base angle $\varphi=63^{\circ}$ and refractive index $n=1.8$ (for $\lambda=510.5 \mathrm{~nm}$ ) and were created according to technique $[9,10]$. The laser based on copper vapour had the following characteristics: the light pulse duration - $30 \mathrm{~ns}$, pulse repetition frequency $-8 \mathrm{kHz}$. LCM was controlled by the sine-form signal with the frequency of $8 \mathrm{kHz}$ and amplitude of $8 \ldots 80 \mathrm{~V}$ modulated by square pulses with the duration of 0.5 to $10 \mathrm{~ms}$. Optical pulses were registered by the coaxial photoelectric cell FEK-16 and oscilloscope I2-7 with the minimal measuring time of $0.5 \mathrm{~ns}$.

\section{Results and discussion}

The results of our experimental investigation of LCM as a controlling element in the resonator channel of LPM shows that it demonstrates switching times of 0.2 and $0.4 \mathrm{~ms}$ (Fig. 3). Switching times were determined at the level 0.9 at increasing and 0.1 at decreasing of electrooptical characteristics of LCM.

The analysis of the oscillogram in Fig. 3 shows that developed LCM can transmit pulse burst with the minimal quantity of pulses equal to 7 . The saturation is characterized by the obvious step that guarantees the transmission of powerful laser pulses with practically stable energy. When control voltage applied to LCM is changed from 30 up to $70 \mathrm{~V}$, it demonstrates the change of transmission coefficient and, as the consequence, the change of laser pulse energy for object treatment in the range of $10 \mathrm{~dB}$. The correspondent oscillograms are shown in Fig. 4. As it can be seen in this figure, decreasing the control voltage not only leads to decreasing the laser pulse power but also to increasing the "switch on" time and to insufficient decreasing the "switch off" time.

Investigations of modulation percentage $M$ and optical losses $P$ show the possibility of obtaining $\mathrm{M}=99 \%$ at $\mathrm{P}=60 \%$. Such high value of optical loses can be explained by the loses in polarizer $(P=50 \%)$ placed on input plane of LCM and spurious reflection of light from

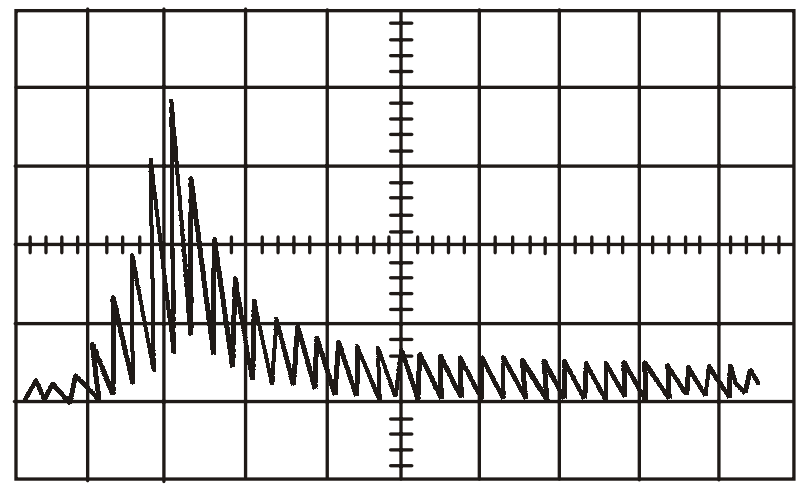

Fig. 3. Oscillogram of an optical signal. The control voltage $50 \mathrm{~V}$, $\tau_{i}=0.5 \mathrm{~ms}$ (time resolution $0.5 \mathrm{~ms} / \mathrm{scale}$ )
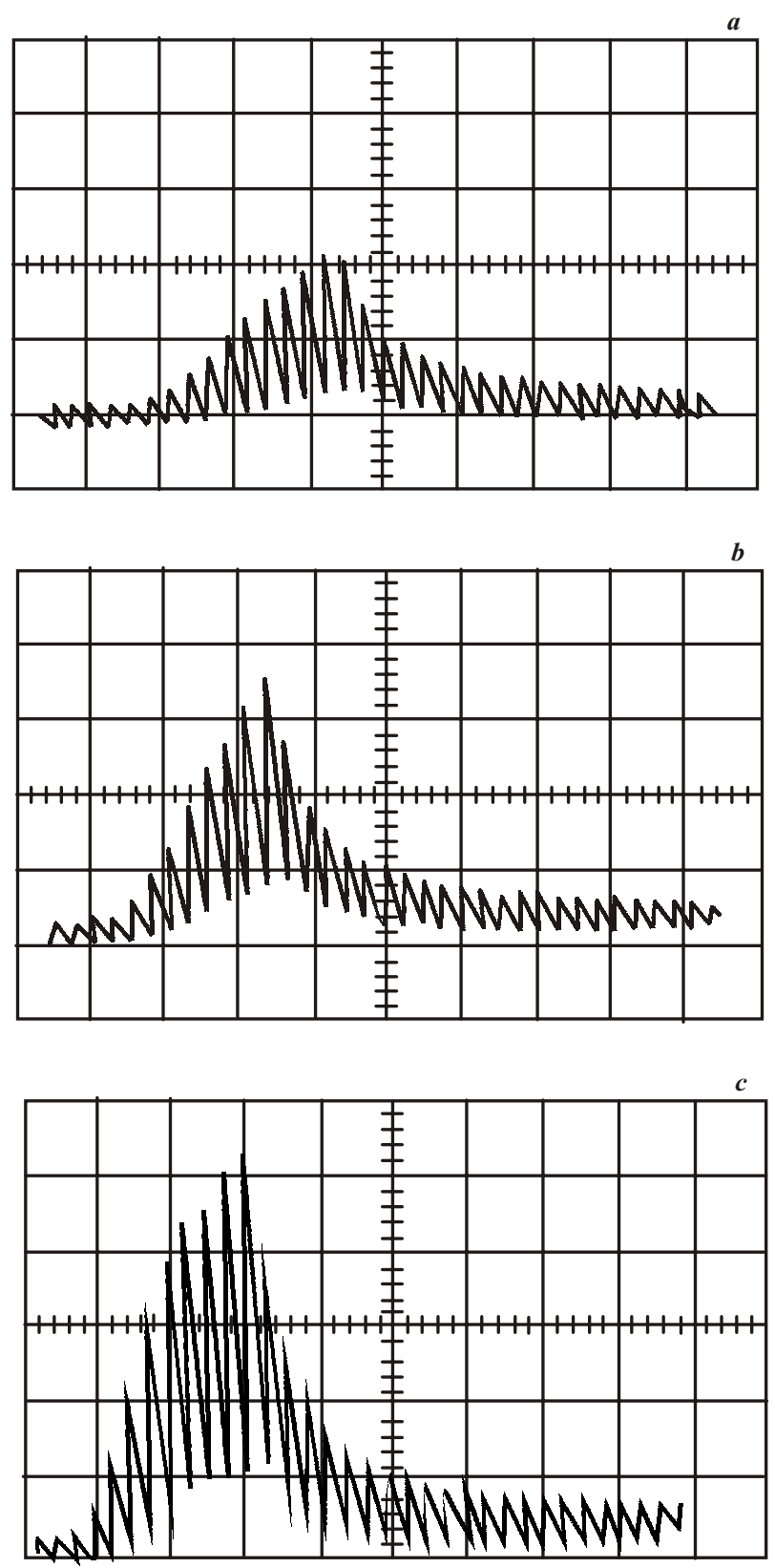

Fig. 4. Oscillograms of optical signals. $\tau_{i}=1 \mathrm{~ms}$, control voltages $30 \mathrm{~V}(a), 40 \mathrm{~V}(b)$ and $60 \mathrm{~V}(c)$ (time resolution $0.5 \mathrm{~ms} / \mathrm{scale}$ ).

interfaces between air and glass, glass and transparent electrode, glass and air. However, almost in all the cases such loses do not affect efficiency and quality of object treatment.

For investigation of LPM with LCM, technological possibilities treatment of different samples were performed.

Thin chromium films (thickness $0.1 \mu \mathrm{m}$ ) deposited on a glass substrate and polysilicide thin films (thickness $0.3 \mu \mathrm{m}$ ) deposited on a substrate from phosphorous silicide glass were used as laboratory samples. The size of light spot was 1.5 by $1.5 \mu \mathrm{m}$. The results of sample treatment shows that using LCM as a light shutter in LPM gives 
possibility to remove chromium from area with reproducible sizes $2 \mu \mathrm{m}$. The layer-by-layer controllable removal of polysilicide thin film was observed when amplitude of LCM control voltage was varying.

\section{Conclusions}

The results of our experimental investigations shows that using LCM based on electrically controlled reflection from the interface between isotropic dielectric and nematic liquid crystal in treatment and observation channels of LPM, which simultaneously acts as a modulator, attenuator and light beam splitter can sufficiently improve characteristics of LPM and enlarge its functional possibilities.

\section{References}

1. J.F. Readly, Industrial Application of Lasers, N.Y.: Academic Prerss, (1978).

2. W.W., Duley Laser Processing and Analysis of Materials, N.Y. and London: Plenum Press (1983).
3. P. Rabinovitz, K. Chimenti // J. Opt. Soc. Am., 60, p. 1577 (1970).

4. K.I. Zemskov, A.A. Isaev, M.A. Kazaryan, G.G. Petrash, Laser projection microscope // Kvantovaya electronika, 1, p.14 (1974).

5. Belayev V.P. i dr. Visual examination plant with laser micro projector // Electronnaya promyshlennost, 5, p. 39-40 (1976).

6. K.I. Zemskov, A.A. Isaev, M.A. Kazaryan, G.G. Petrash, The means of a treatment, registration and observation with the help super radiant laser medium, International request PCT/SU/ 80/ 00141.

7. R. Zelinski, I. Karpov, A. Skripnicchenko, V.M. Sorokin, LC-modulator in the channel of laser projection microscope I V kn.: Optoelektronnye metody i sredstva obrabotki izobrajeniy, Vinniza-Tbilissi, p. 379-381 (1987).

8. P.F. Oleksenko, V.M. Sorokin, R.Ya. Zelinskyy, The light modulation in the isotropic dielectric - single axis LC interface // J. prikladnoy spektroscopii, 43(5), p. 866 (1985).

9. P.F. Oleksenko, V.M. Sorokin, R.Ya. Zelinskyy, Investigation of electro-optical characteristics of the isotropic dielectric - LC interface // Optoelectronica i poluprovodnicovaya tehnica, 10, p. 54-58 (1986).

10. P.F. Oleksenko, V.M. Sorokin, R.Ya. Zelinskyy Electrooptic Glass-NLC Light Modulator // Proc. SPIE, 2372, p. 323-329 (1995). 